\title{
Algebraic Techniques for Linear Equations over Quaternions and Split Quaternions: A Unified Approach in Quaternionic and Split Quaternionic Mechanics
}

\author{
Gang Wang1,2, Zhenwei Guo1, Dong Zhang3, Tongsong Jiang1,2,3* \\ ${ }^{1}$ School of Mathematical Science, Liaocheng University, Liaocheng, China \\ ${ }^{2}$ School of Mathematics and Statistics, Heze University, Heze, China \\ ${ }^{3}$ College of Mathematics and Systems Science, Shandong University of Science and Technology, Qingdao, China \\ Email: wang_gang93@163.com, ^jiangtongsong@sina.com,dz_zhangdong@sina.com
}

How to cite this paper: Wang, G., Guo, Z.W., Zhang, D. and Jiang, T.S. (2019) Algebraic Techniques for Linear Equations over Quaternions and Split Quaternions: A Unified Approach in Quaternionic and Split Quaternionic Mechanics. Journal of Applied Mathematics and Physics, 7, 1718-1731.

https://doi.org/10.4236/jamp.2019.78118

Received: July 18, 2019

Accepted: August 13, 2019

Published: August 16, 2019

Copyright $\odot 2019$ by author(s) and Scientific Research Publishing Inc. This work is licensed under the Creative Commons Attribution International License (CC BY 4.0).

http://creativecommons.org/licenses/by/4.0/

\begin{abstract}
This paper aims to present, in a unified manner, algebraic techniques for linear equations which are valid on both the algebras of quaternions and split quaternions. This paper, introduces a concept of v-quaternion, studies the problem of $\mathrm{v}$-quaternionic linear equations by means of a complex representation and a real representation of $\mathrm{v}$-quaternion matrices, and gives two algebraic methods for solving v-quaternionic linear equations. This paper also gives a unification of algebraic techniques for quaternionic and split quaternionic linear equations in quaternionic and split quaternionic mechanics.
\end{abstract}

\section{Keywords}

V-Quaternion, Complex Representation, Real Representation, Linear Equations, Quaternion, Split Quaternion

\section{Introduction}

A quaternion, which was found in 1840 by William Rowan Hamilton [1], is in the form of $q=q_{1}+q_{2} \mathrm{i}+q_{3} \mathrm{j}+q_{4} \mathrm{k}, \quad \mathrm{i}^{2}=\mathrm{j}^{2}=\mathrm{k}^{2}=-1, \quad \mathrm{ijk}=-1$, where $q_{1}, q_{2}, q_{3}, q_{4} \in \mathbf{R}$, and $\mathrm{ij}=-\mathrm{ji}=\mathrm{k}, \mathrm{jk}=-\mathrm{kj}=\mathrm{i}, \mathrm{ki}=-\mathrm{ik}=\mathrm{j}$. Quaternion algebra has been playing a significant role recently in geometric and physical applications, many geometric problems can be represented by quaternions. In paper [2], the authors showed that a unit timelike quaternion represents a rotation in the Minkowski 3 space, and expressed Lorentzian rotation matrix generated 
with a time like quaternion. In paper [3], the authors studied the problem of using quaternions in unconstrained nonlinear optimization of 3-D rotations, and gave an easy and accurate method for applying the quaternion representation of 3-D rotations.

A split quaternion (or coquaternion), which was found in 1849 by James Cockle [4], is in the form of $q=q_{1}+q_{2} \mathrm{i}+q_{3} \mathrm{j}+q_{4} \mathrm{k}, \mathrm{i}^{2}=-1, \mathrm{j}^{2}=\mathrm{k}^{2}=1$, $\mathrm{ijk}=1$, where $q_{1}, q_{2}, q_{3}, q_{4} \in \mathbf{R}$, and $\mathrm{ij}=-\mathrm{ji}=\mathrm{k}, \mathrm{jk}=-\mathrm{kj}=-\mathrm{i}, \quad \mathrm{ki}=-\mathrm{ik}=\mathrm{j}$ and denotes the sets of quaternions and split quaternions respectively by $\mathbf{H}$ and $\mathbf{H}_{s}$. The quaternion ring $\mathbf{H}$ and the split quaternion ring $\mathbf{H}_{s}$ are two associative and noncommutative 4-dimensional Clifford algebras, and the split quaternion ring $\mathbf{H}_{s}$ contains zero divisors, nilpotent elements and nontrivial idempotents. In paper [5], the authors stated the rotations in Minkowski 3 space by split quaternions. In paper [6], the authors studied dual split quaternions and screw motion in 3-dimensional Lorentzian space, and obtained the components of a dual split quaternion by replacing the L-Euler parameters with their split dual versions. In paper [7], the authors studied eigenvalue problem of a rotation matrix in Minkowski 3 space by using split quaternions, and gave the characterizations of eigenvalues of a rotation matrix in Minkowski 3 space according to only first component of the corresponding quaternion. Quaternions and split quaternions in the study of geometry and physic are more than those, e.g. [8]-[13].

A v-quaternion is in the form of

$$
q=q_{1}+q_{2} \mathrm{i}+q_{3} \mathrm{j}+q_{4} \mathrm{k}, \mathrm{i}^{2}=-1, \mathrm{j}^{2}=v, \mathrm{ij}=-\mathrm{ji}=\mathrm{k},
$$

in which $0 \neq v \in \mathbf{R}, \quad q_{1}, q_{2}, q_{3}, q_{4} \in \mathbf{R}$, and $\mathrm{k}^{2}=\mathrm{ijk}=v, \quad \mathrm{jk}=-\mathrm{kj}=-v \mathrm{i}$, $\mathrm{ik}=-\mathrm{ki}=-\mathrm{j}$. Let $\mathbf{H}_{v}$ denote the set of v-quaternion. Obviously, the set of all v-quaternion is also a noncommutative 4-dimensional Clifford algebra. Specially, when $v=-1$, the ring of the v-quaternion $\mathbf{H}_{v}$ is the ring of the quaternion $\mathbf{H}$; when $v=1$, the ring of the $\mathbf{v}$-quaternion $\mathbf{H}_{v}$ is the ring of the split quaternion $\mathbf{H}_{s}$.

In the geometry research and physical application of quaternion and split quaternion, the problems of solving quaternionic and split quaternionic equations are often encountered. In paper [14], by means of a complex representation of quaternion matrices, the authors studied the problems of quaternionic linear equations, and gave an algorithm for quaternionic linear equations. In paper [15], by means of a complex representation and a real representation of split quaternion matrices, the authors studied the split quaternionic least squares problem, and derived two algebraic methods for finding solutions of the problems in split quaternionic mechanics. For the problems of quaternions and split quaternions, the scholars need to discuss by classification. However, as two special cases of four-dimensional algebra, it is of theoretical and practical significance to solve them in a unified way. This paper aims to present, in a unified manner, algebraic techniques for linear equations which are valid on both the algebras of quaternions and split quaternions. This paper, by means of a complex represen- 
tation and a real representation of v-quaternion matrices, studies the problem of $\mathrm{v}$-quaternionic linear equations, and gives two algebraic methods for solving v-quaternionic linear equations. This paper also gives a unification of algebraic techniques for quaternionic and split quaternionic linear equations in quaternionic and split quaternionic mechanics.

Let $\mathbf{R}$ be the real number field, $\mathbf{C}=\mathbf{R} \oplus \mathbf{R i}$ the complex number field. If $q=q_{1}+q_{2} \mathrm{i}+q_{3} \mathrm{j}+q_{4} \mathrm{k} \in \mathbf{H}_{v}, \quad \bar{q}=q_{1}-q_{2} \mathrm{i}-q_{3} \mathrm{j}-q_{4} \mathrm{k}$ is the conjugate of $q$. For any matrix $A=\left(a_{s t}\right) \in \mathbf{H}_{v}^{m \times n}, \quad \bar{A}=\left(\bar{a}_{s t}\right), A^{\mathrm{T}}=\left(a_{t s}\right), \quad A^{*}=\left(\bar{a}_{t s}\right), A^{-1}$ denote the conjugate, the transpose, the conjugate transpose and the inverse of the ma$\operatorname{trix} A$, respectively.

This paper is organized as follows. In Section 2, we give two new matrix representations of v-quaternion matrix, and discuss some properties and conclusions of complex representation and real representation of $\mathrm{v}$-quaternion matrices. In Section 3, we present the complex representation method for solving v-quaternionic linear equations and some numerical examples. In Section 4, we present the real representation method for solving v-quaternionic linear equations and some numerical examples. In Section 5, we summarize this paper.

\section{Complex Representation and Real Representation of V-Quaternion Matrices}

For any v-quaternion matrix $A=A_{1}+A_{2} \mathrm{i}+A_{3} \mathrm{j}+A_{4} \mathrm{k}=\left(A_{1}+A_{2} \mathrm{i}\right)+\left(A_{3}+A_{4} \mathrm{i}\right) \mathrm{j}=B_{1}+B_{2} \mathrm{j} \in \mathbf{H}_{v}^{m \times n}$, $A_{1}, A_{2}, A_{3}, A_{4} \in \mathbf{R}^{m \times n}, B_{1}, B_{2} \in \mathbf{C}^{m \times n}$, the complex representation $A^{C}$ of the $\mathrm{v}$-quaternion matrix $A$ is defined to be

$$
A^{C}=\left[\begin{array}{cc}
B_{1} & v B_{2} \\
\bar{B}_{2} & \bar{B}_{1}
\end{array}\right],
$$

and the real representation $A^{R}$ of the v-quaternion matrix $A$ is defined to be

$$
A^{R}=\left[\begin{array}{cccc}
A_{1} & -A_{2} & v A_{3} & v A_{4} \\
A_{2} & A_{1} & v A_{4} & -v A_{3} \\
A_{3} & A_{4} & A_{1} & -A_{2} \\
A_{4} & -A_{3} & A_{2} & A_{1}
\end{array}\right] .
$$

For any v-quaternion matrix $A, B \in \mathbf{H}_{v}^{m \times n}, C \in \mathbf{H}_{v}^{n \times p}, a \in \mathbf{R}$, for $\sigma \in\{C, R\}$, it is easy to prove the following equalities by direct calculation.

$$
(A+B)^{\sigma}=A^{\sigma}+B^{\sigma},(a A)^{\sigma}=a A^{\sigma},(A C)^{\sigma}=A^{\sigma} C^{\sigma},
$$

and

$$
Q_{m}^{-1} A^{C} Q_{n}=\overline{A^{C}}
$$

where $Q_{t}=\left[\begin{array}{cc}0 & v I_{t} \\ I_{t} & 0\end{array}\right]$.

Similarly, by direct calculation we get the following results.

$$
P_{m}^{-1} A^{R} P_{n}=A^{R}, R_{m}^{-1} A^{R} R_{n}=A^{R}, S_{m}^{-1} A^{R} S_{n}=A^{R},
$$


where $P_{t}=\left[\begin{array}{cccc}0 & -I_{t} & 0 & 0 \\ I_{t} & 0 & 0 & 0 \\ 0 & 0 & 0 & I_{t} \\ 0 & 0 & -I_{t} & 0\end{array}\right], R_{t}=\left[\begin{array}{cccc}0 & 0 & v I_{t} & 0 \\ 0 & 0 & 0 & v I_{t} \\ I_{t} & 0 & 0 & 0 \\ 0 & I_{t} & 0 & 0\end{array}\right]$,
$S_{t}=\left[\begin{array}{cccc}0 & 0 & 0 & v I_{t} \\ 0 & 0 & -v I_{t} & 0 \\ 0 & -I_{t} & 0 & 0 \\ I_{t} & 0 & 0 & 0\end{array}\right]$, and $P_{t}^{-1}=-P_{t}, R_{t}^{-1}=\frac{1}{v} R_{t}, S_{t}^{-1}=\frac{1}{v} S_{t}$.

Lemma 2.1 For two special cases of quaternion $(v=-1)$ and split quaternion $(v=1)$ matrices, clearly by (2.1) and (2.2) the complex representation and the real representation are respectively to be

$$
\begin{aligned}
A^{C}=\left[\begin{array}{cc}
B_{1} & -B_{2} \\
\bar{B}_{2} & \bar{B}_{1}
\end{array}\right], A^{R}=\left[\begin{array}{cccc}
A_{1} & -A_{2} & -A_{3} & -A_{4} \\
A_{2} & A_{1} & -A_{4} & A_{3} \\
A_{3} & A_{4} & A_{1} & -A_{2} \\
A_{4} & -A_{3} & A_{2} & A_{1}
\end{array}\right], A \in \mathbf{H}^{m \times n} . \\
A^{C}=\left[\begin{array}{cc}
B_{1} & B_{2} \\
\bar{B}_{2} & \bar{B}_{1}
\end{array}\right], A^{R}=\left[\begin{array}{cccc}
A_{1} & -A_{2} & A_{3} & A_{4} \\
A_{2} & A_{1} & A_{4} & -A_{3} \\
A_{3} & A_{4} & A_{1} & -A_{2} \\
A_{4} & -A_{3} & A_{2} & A_{1}
\end{array}\right], A \in \mathbf{H}_{s}^{m \times n} .
\end{aligned}
$$

For any v-quaternion matrix $A \in \mathbf{H}_{v}^{m \times n}$, the $\operatorname{rank} \operatorname{rank}(A)$ of the matrix $A$ is defined to be

$$
\operatorname{rank}(A) \equiv \frac{1}{2} \operatorname{rank}\left(A^{C}\right),
$$

or

$$
\operatorname{rank}(A) \equiv \frac{1}{4} \operatorname{rank}\left(A^{R}\right) .
$$

By the definition of rank and (2.3), it is easy to get the following results by direct calculation. If $A, B \in \mathbf{H}_{v}^{m \times n}, C \in \mathbf{H}_{v}^{n \times p}$, then $\operatorname{rank}(A+B) \leq \operatorname{rank}(A)+\operatorname{rank}(B)$ and $\operatorname{rank}(A C) \leq \min \{\operatorname{rank}(A), \operatorname{rank}(C)\}$.

\section{Algebraic Method of Complex Representation}

If $A \in \mathbf{H}_{v}^{m \times n}, B \in \mathbf{H}_{v}^{m \times p}$, then by the definition of complex representation and (2.3), $A X=B$ if and only if $A^{C} X^{C}=B^{C}$. That is $A X=B$ has a solution $X$ if and only if $A^{C} Y=B^{C}$ has a solution $Y=X^{C}$.

Theorem 3.1 For $A \in \mathbf{H}_{v}^{m \times n}, B \in \mathbf{H}_{v}^{m \times p}$. Then

1) V-quaternionic linear equations $A X=B$ have a solution if and only if $\operatorname{rank}(A)=\operatorname{rank}(A, B)$, i.e. $A X=B$ has a solution if and only if $A^{C} Y=B^{C}$ has a solution, and if $\operatorname{rank}(A)=\operatorname{rank}(A, B)=n$, then v-quaternionic linear equations $A X=B$ have a unique solution.

2) If $Y$ is a solution to $A^{C} Y=B^{C}$, then the following v-quaternion matrix is a solution to $A X=B$, 


$$
X=\frac{1}{4}\left[I_{n}, I_{n} \mathrm{j}\right]\left(Y+Q_{n}^{-1} \bar{Y} Q_{p}\right)\left[\begin{array}{c}
I_{p} \\
\frac{1}{v} I_{p} \mathrm{j}
\end{array}\right],
$$

in which $Q_{t}=\left[\begin{array}{cc}0 & v I_{t} \\ I_{t} & 0\end{array}\right]$.

Proof: If $Y$ is a solution of $A^{C} Y=B^{C}$, by (2.4),

$$
A^{C} Y=B^{C} \Leftrightarrow \overline{A^{C}}\left(Q_{n}^{-1} Y Q_{p}\right)=\overline{B^{C}} \Leftrightarrow A^{C}\left(Q_{n}^{-1} \bar{Y} Q_{p}\right)=B^{C},
$$

i.e. $Q_{n}^{-1} \bar{Y} Q_{p}$ is a solution of $A^{C} Y=B^{C}$, therefore

$$
\hat{Y}=\frac{1}{2}\left(Y+Q_{n}^{-1} \bar{Y} Q_{p}\right)
$$

is also a solution of $A^{C} Y=B^{C}$. Let

$$
Y=\left[\begin{array}{ll}
Z_{11} & z_{12} \\
z_{21} & z_{22}
\end{array}\right] \in \mathbf{C}^{2 n \times 2 p}, z_{t s} \in \mathbf{C}^{n \times p}, s, t=1,2 .
$$

It is easy to get, by direct calculation,

$$
\hat{Y}=\left[\begin{array}{cc}
\hat{z}_{1} & v \hat{z}_{2} \\
\hat{\mathrm{z}}_{2} & \overline{\mathrm{z}}_{1}
\end{array}\right] \in \mathbf{C}^{2 n \times 2 p},
$$

in which

$$
\hat{z}_{1}=\frac{1}{2}\left(z_{11}+\bar{z}_{22}\right), \hat{z}_{2}=\frac{1}{2}\left(\frac{1}{v} z_{12}+\bar{z}_{21}\right) .
$$

By (3.5), we construct a v-quaternion matrix.

$$
X=\hat{z}_{1}+\hat{z}_{2} \mathrm{j}=\frac{1}{2}\left[I_{n}, I_{n} \mathrm{j}\right] \hat{Y}\left[\begin{array}{c}
I_{p} \\
\frac{1}{V} I_{p} \mathrm{j}
\end{array}\right] .
$$

Clearly $X^{C}=\hat{Y}$. This means that $X^{C}=\hat{Y}$ is a solution of $A^{C} Y=B^{C}$, so $X$ is a solution of $A X=B$.

From the statement above we get following results. When the v-quaternionic linear equations $A X=B$ have a solution, we can find a solution by a solution of complex representation equation $A^{C} Y=B^{C}$ from the formula (3.1).

The following two special cases about quaternions and split quaternions come from Theorem 3.1 respectively with $v=-1$ and $v=1$.

Corollary 3.2 For $A \in \mathbf{H}^{m \times n}, B \in \mathbf{H}^{m \times p}$. Then

1) The quaternionic linear equations $A X=B$ have a solution if and only if $\operatorname{rank}(A)=\operatorname{rank}(A, B)$, i.e. $A X=B$ has a solution if and only if $A^{C} Y=B^{C}$ has a solution, and if $\operatorname{rank}(A)=\operatorname{rank}(A, B)=n$, then quaternionic linear equations $A X=B$ have a unique solution.

2) If $Y$ is a solution to $A^{C} Y=B^{C}$, then the following quaternion matrix is a solution to $A X=B$,

$$
X=\frac{1}{4}\left[I_{n}, I_{n} \mathrm{j}\right]\left(Y+Q_{n}^{-1} \bar{Y} Q_{p}\right)\left[\begin{array}{c}
I_{p} \\
-I_{p} \mathrm{j}
\end{array}\right],
$$


in which $Q_{t}=\left[\begin{array}{cc}0 & -I_{t} \\ I_{t} & 0\end{array}\right]$.

Corollary 3.3 For $A \in \mathbf{H}_{s}^{m \times n}, B \in \mathbf{H}_{s}^{m \times p}$. Then

1) The split quaternionic linear equations $A X=B$ have a solution if and only if $\operatorname{rank}(A)=\operatorname{rank}(A, B)$, i.e. $A X=B$ has a solution if and only if $A^{C} Y=B^{C}$ has a solution, and if $\operatorname{rank}(A)=\operatorname{rank}(A, B)=n$, then split quaternionic linear equations $A X=B$ have a unique solution.

2) If $Y$ is a solution to $A^{C} Y=B^{C}$, then the following split quaternion matrix is a solution to $A X=B$,

$$
X=\frac{1}{4}\left[I_{n}, I_{n} \mathrm{j}\right]\left(Y+Q_{n}^{-1} \bar{Y} Q_{p}\right)\left[\begin{array}{c}
I_{p} \\
I_{p} \mathrm{j}
\end{array}\right]
$$

in which $Q_{t}=\left[\begin{array}{cc}0 & I_{t} \\ I_{t} & 0\end{array}\right]$.

In the similarly way, we have the following result.

Theorem 3.4 For $A \in \mathbf{H}_{v}^{m \times n}, C \in \mathbf{H}_{v}^{p \times q}, B \in \mathbf{H}_{v}^{m \times q}$. Then

1) $\mathrm{V}$-quaternionic matrix equation $A X C=B$ has a solution if and only if $\operatorname{rank}(A)=\operatorname{rank}(A, B)$ and $\operatorname{rank}(C)=\operatorname{rank}\left(\begin{array}{l}C \\ B\end{array}\right)$, i.e. $A X C=B$ has a solution if and only if $A^{C} Y C^{C}=B^{C}$ has a solution, and if $\operatorname{rank}(A)=\operatorname{rank}(A, B)=n$, $\operatorname{rank}(C)=\operatorname{rank}\left(\begin{array}{l}C \\ B\end{array}\right)=p$, then v-quaternionic matrix equation $A X C=B$ has a unique solution.

2) If $Y$ is a solution to $A^{C} Y C^{C}=B^{C}$, then the following v-quaternion matrix is a solution to $A X C=B$,

$$
X=\frac{1}{4}\left[I_{n}, I_{n} \mathrm{j}\right]\left(Y+Q_{n}^{-1} \bar{Y} Q_{p}\right)\left[\begin{array}{c}
I_{p} \\
\frac{1}{v} I_{p} \mathrm{j}
\end{array}\right],
$$

in which $Q_{t}=\left[\begin{array}{cc}0 & v I_{t} \\ I_{t} & 0\end{array}\right]$.

The proof process is similar to the Theorem 3.1.

Remark 1 The above theorems and corollaries not only give the necessary and sufficient conditions for quaternion and split quaternion matrix equations $A X=B, A X C=B$ to have a solution, but also a unification of representation for a solution.

Example 3.1

Let

$$
A=\left(\begin{array}{cc}
\mathrm{i} & 1+\mathrm{j} \\
-1+\mathrm{j} & -\mathrm{k}
\end{array}\right) \text { and } B=\left(\begin{array}{c}
\mathrm{i} \\
-1
\end{array}\right) \text {. }
$$

Find all solutions of the v-quaternionic linear equations $A X=B$.

By the complex representation of the v-quaternion matrix, we know 


$$
A^{C}=\left[\begin{array}{cccc}
\mathrm{i} & 1 & 0 & v \\
-1 & 0 & v & -v \mathrm{i} \\
0 & 1 & -\mathrm{i} & 1 \\
1 & \mathrm{i} & -1 & 0
\end{array}\right], B^{C}=\left[\begin{array}{cc}
\mathrm{i} & 0 \\
-1 & 0 \\
0 & -\mathrm{i} \\
0 & -1
\end{array}\right],
$$

and if $v \neq \frac{7 \pm \sqrt{45}}{2}$, then $\operatorname{rank}\left(A^{C}\right)=\operatorname{rank}\left(A^{C}, B^{C}\right)=4$, i.e.

$\operatorname{rank}(A)=\operatorname{rank}(A, B)=2$, then the v-quaternionic linear equations $A X=B$ have a unique solution.

For the matrix equation $A^{C} Y=B^{C}$, the unique solution is easily found to be

$$
Y=\left[\begin{array}{cc}
\frac{-5 v+1}{v^{2}-7 v+1} & \frac{v(1-2 v)}{v^{2}-7 v+1} \\
\frac{-3 v}{v^{2}-7 v+1} \mathrm{i} & \frac{v(-1-v)}{v^{2}-7 v+1} \mathrm{i} \\
\frac{1-2 v}{v^{2}-7 v+1} & \frac{-5 v+1}{v^{2}-7 v+1} \\
\frac{1+v}{v^{2}-7 v+1} \mathrm{i} & \frac{3 v}{v^{2}-7 v+1} \mathrm{i}
\end{array}\right] .
$$

By (3.1), we easily find the unique solution $X$ of v-quaternionic linear equations $A X=B$, and

$$
\begin{aligned}
X & =\frac{1}{4}\left[I_{2}, I_{2} \mathrm{j}\right]\left(Y+Q_{n}^{-1} \bar{Y} Q_{p}\right)\left[\begin{array}{c}
1 \\
\frac{1}{v} \mathrm{j}
\end{array}\right] \\
& =\left[\frac{-5 v+1}{v^{2}-7 v+1}+\frac{1-2 v}{v^{2}-7 v+1} \mathrm{j} \frac{-3 v}{v^{2}-7 v+1} \mathrm{i}-\frac{1+v}{v^{2}-7 v+1} \mathrm{k}\right]^{\mathrm{T}} .
\end{aligned}
$$

The following two examples are special cases of the above conclusion.

Case 1: For quaternionic linear equations $A X=B$ with $v=-1$. It is easy to know $A^{C}$ and $B^{C}$ by (2.6a),

$$
A^{C}=\left[\begin{array}{cccc}
\mathrm{i} & 1 & 0 & -1 \\
-1 & 0 & -1 & \mathrm{i} \\
0 & 1 & -\mathrm{i} & 1 \\
1 & \mathrm{i} & -1 & 0
\end{array}\right], B^{C}=\left[\begin{array}{cc}
\mathrm{i} & 0 \\
-1 & 0 \\
0 & -\mathrm{i} \\
0 & -1
\end{array}\right]
$$

and $\operatorname{rank}\left(A^{C}\right)=\operatorname{rank}\left(A^{C}, B^{C}\right)=4$. Clearly, the linear equations $A^{C} Y=B^{C}$ have a unique solution. The unique solution is easily found to be

$$
Y=\left[\begin{array}{cc}
\frac{2}{3} & -\frac{1}{3} \\
\frac{1}{3} \mathrm{i} & 0 \\
\frac{1}{3} & \frac{2}{3} \\
0 & -\frac{1}{3} \mathrm{i}
\end{array}\right] .
$$

By (3.8), we easily find the unique solution $X$ of quaternionic linear equations 
$A X=B$, and

$$
X=\frac{1}{4}\left[I_{2}, I_{2} \mathrm{j}\right]\left(Y+Q_{n}^{-1} \bar{Y} Q_{p}\right)\left[\begin{array}{c}
1 \\
-\mathrm{j}
\end{array}\right]=\left[\begin{array}{cc}
\frac{2}{3}+\frac{1}{3} \mathrm{j} & \frac{1}{3} \mathrm{i}
\end{array}\right]^{\mathrm{T}} .
$$

Case 2: For split quaternionic linear equations $A X=B$ with $v=1$. It is easy to know $A^{C}$ and $B^{C}$ by (2.6b),

$$
A^{C}=\left[\begin{array}{cccc}
\mathrm{i} & 1 & 0 & 1 \\
-1 & 0 & 1 & -\mathrm{i} \\
0 & 1 & -\mathrm{i} & 1 \\
1 & \mathrm{i} & -1 & 0
\end{array}\right], B^{C}=\left[\begin{array}{cc}
\mathrm{i} & 0 \\
-1 & 0 \\
0 & -\mathrm{i} \\
0 & -1
\end{array}\right]
$$

and $\operatorname{rank}\left(A^{C}\right)=\operatorname{rank}\left(A^{C}, B^{C}\right)=4$. Clearly, the linear equations $A^{C} Y=B^{C}$ have a unique solution. The unique solution is easily found to be

$$
Y=\left[\begin{array}{cc}
\frac{4}{5} & \frac{1}{5} \\
\frac{3}{5} \mathrm{i} & \frac{2}{5} \mathrm{i} \\
\frac{1}{5} & \frac{4}{5} \\
-\frac{2}{5} \mathrm{i} & -\frac{3}{5} \mathrm{i}
\end{array}\right] .
$$

By (3.9), we easily find the unique solution $X$ of split quaternionic linear equations $A X=B$, and

$$
X=\frac{1}{4}\left[I_{2}, I_{2} \mathrm{j}\right]\left(Y+Q_{n}^{-1} \bar{Y} Q_{p}\right)\left[\begin{array}{c}
1 \\
\mathrm{j}
\end{array}\right]=\left[\frac{4}{5}+\frac{1}{5} \mathrm{j} \quad \frac{3}{5} \mathrm{i}+\frac{2}{5} \mathrm{k}\right]^{\mathrm{T}} .
$$

\section{Algebraic Method of Real Representation}

If $A \in \mathbf{H}_{v}^{m \times n}, B \in \mathbf{H}_{v}^{m \times p}$, then by the definition of real representation, $A X=B$ if and only if $A^{R} X^{R}=B^{R}$. That is $A X=B$ has a solution $X$ if and only if $A^{R} Y=B^{R}$ has a solution $Y=X^{R}$.

Theorem 4.1 For $A \in \mathbf{H}_{v}^{m \times n}, B \in \mathbf{H}_{v}^{m \times p}$. Then

1) $\mathrm{V}$-quaternionic linear equations $A X=B$ have a solution if and only if $\operatorname{rank}(A)=\operatorname{rank}(A, B)$, i.e. $A X=B$ has a solution if and only if $A^{R} Y=B^{R}$ has a solution, and if $\operatorname{rank}(A)=\operatorname{rank}(A, B)=n$, then v-quaternionic linear equations $A X=B$ have a unique solution.

2) If $Y$ is a solution to $A^{R} Y=B^{R}$, then the following v-quaternion matrix is a solution to $A X=B$,

$$
X=\frac{1}{16}\left[I_{n}, I_{n} \mathrm{i}, I_{n} \mathrm{j}, I_{n} \mathrm{k}\right]\left(Y-P_{n} Y P_{p}+\frac{1}{v} R_{n} Y R_{p}+\frac{1}{V} S_{n} Y S_{p}\right)\left[\begin{array}{c}
I_{p} \\
-I_{p} \mathrm{i} \\
\frac{1}{v} I_{p} \mathrm{j} \\
\frac{1}{v} I_{p} \mathrm{k}
\end{array}\right] .
$$

Proof: If $Y$ is a solution of $A^{R} Y=B^{R}$, by (2.5), 


$$
A^{R} Y=B^{R} \Leftrightarrow P_{m}^{-1} A^{R} P_{n}\left(P_{n}^{-1} Y P_{p}\right)=P_{m}^{-1} B^{R} P_{p} \Leftrightarrow A^{R}\left(P_{n}^{-1} Y P_{p}\right)=B^{R},
$$

i.e. $P_{n}^{-1} Y P_{p}$ is a solution of $A^{R} Y=B^{R}$. Similarly, $R_{n}^{-1} Y R_{p}, S_{n}^{-1} Y S_{p}$ are also solution of $A^{R} Y=B^{R}$.

$$
\begin{aligned}
\hat{Y} & =\frac{1}{4}\left(Y+P_{n}^{-1} Y P_{p}+R_{n}^{-1} Y R_{p}+S_{n}^{-1} Y S_{p}\right) \\
& =\frac{1}{4}\left(Y-P_{n} Y P_{p}+\frac{1}{V} R_{n} Y R_{p}+\frac{1}{V} S_{n} Y S_{p}\right)
\end{aligned}
$$

is also a solution of $A^{R} Y=B^{R}$. Let

$$
Y=\left[\begin{array}{cccc}
z_{11} & z_{12} & z_{13} & z_{14} \\
z_{21} & z_{22} & z_{23} & z_{24} \\
z_{31} & z_{32} & z_{33} & z_{34} \\
z_{41} & z_{42} & z_{43} & z_{44}
\end{array}\right] \in \mathbf{R}^{4 n \times 4 p}, z_{t s} \in \mathbf{R}^{n \times p}, s, t=1,2,3,4 .
$$

It is easy to get, by direct calculation,

$$
\hat{Y}=\left[\begin{array}{cccc}
\hat{z}_{1} & -\hat{z}_{2} & v \hat{z}_{3} & v \hat{z}_{4} \\
\hat{z}_{2} & \hat{z}_{1} & v \hat{z}_{4} & -v \hat{z}_{3} \\
\hat{z}_{3} & \hat{z}_{4} & \hat{z}_{1} & -\hat{z}_{2} \\
\hat{z}_{4} & -\hat{z}_{3} & \hat{z}_{2} & \hat{z}_{1}
\end{array}\right] \in \mathbf{R}^{4 n \times 4 p}
$$

in which

$$
\begin{gathered}
\hat{z}_{1}=\frac{1}{4}\left(z_{11}+z_{22}+z_{33}+z_{44}\right), \hat{z}_{2}=\frac{1}{4}\left(z_{21}-z_{12}+z_{43}-z_{34}\right), \\
\hat{z}_{3}=\frac{1}{4}\left(z_{31}-z_{42}+\frac{1}{v} z_{13}-\frac{1}{v} z_{24}\right), \hat{z}_{4}=\frac{1}{4}\left(z_{41}+z_{32}+\frac{1}{v} z_{23}+\frac{1}{v} z_{14}\right) .
\end{gathered}
$$

By (4.5), we construct a v-quaternion matrix.

$$
X=\hat{z}_{1}+\hat{z}_{2} \mathrm{i}+\hat{z}_{3} \mathrm{j}+\hat{z}_{4} \mathrm{k}=\frac{1}{4}\left[I_{n}, I_{n} \mathrm{i}, I_{n} \mathrm{j}, I_{n} \mathrm{k}\right] \hat{Y}\left[\begin{array}{c}
I_{p} \\
-I_{p} \mathrm{i} \\
\frac{1}{v} I_{p} \mathrm{j} \\
\frac{1}{v} I_{p} \mathrm{k}
\end{array}\right] .
$$

Clearly $X^{R}=\hat{Y}$. This means that $X^{R}=\hat{Y}$ is a solution of $A^{R} Y=B^{R}$, so $X$ is a solution of $A X=B$.

From the statement above we get following results.

The following two special cases about quaternions and split quaternions come from Theorem 4.1 respectively with $v=-1$ and $v=1$.

Corollary 4.2 For $A \in \mathbf{H}^{m \times n}, B \in \mathbf{H}^{m \times p}$. Then

1) The quaternionic linear equations $A X=B$ have a solution if and only if $\operatorname{rank}(A)=\operatorname{rank}(A, B)$, i.e. $A X=B$ has a solution if and only if $A^{R} Y=B^{R}$ has a solution, and if $\operatorname{rank}(A)=\operatorname{rank}(A, B)=n$, then quaternionic linear equations $A X=B$ have a unique solution.

2) If $Y$ is a solution to $A^{R} Y=B^{R}$, then the following quaternion matrix is a solution to $A X=B$, 


$$
X=\frac{1}{16}\left[I_{n}, I_{n} \mathrm{i}, I_{n} \mathrm{j}, I_{n} \mathrm{k}\right]\left(Y-P_{n} Y P_{p}-R_{n} Y R_{p}-S_{n} Y S_{p}\right)\left[\begin{array}{c}
I_{p} \\
-I_{p} \mathrm{i} \\
-I_{p} \mathrm{j} \\
-I_{p} \mathrm{k}
\end{array}\right]
$$

Corollary 4.3 For $A \in \mathbf{H}_{s}^{m \times n}, B \in \mathbf{H}_{s}^{m \times p}$. Then

1) The split quaternionic linear equations $A X=B$ have a solution if and only if $\operatorname{rank}(A)=\operatorname{rank}(A, B)$, i.e. $A X=B$ has a solution if and only if $A^{R} Y=B^{R}$ has a solution, and if $\operatorname{rank}(A)=\operatorname{rank}(A, B)=n$, then split quaternionic linear equations $A X=B$ have a unique solution.

2) If $Y$ is a solution to $A^{R} Y=B^{R}$, then the following split quaternion matrix is a solution to $A X=B$,

$$
X=\frac{1}{16}\left[I_{n}, I_{n} \mathrm{i}, I_{n} \mathrm{j}, I_{n} \mathrm{k}\right]\left(Y-P_{n} Y P_{p}+R_{n} Y R_{p}+S_{n} Y S_{p}\right)\left[\begin{array}{c}
I_{p} \\
-I_{p} \mathrm{i} \\
I_{p} \mathrm{j} \\
I_{p} \mathrm{k}
\end{array}\right] .
$$

In the similarly way, we have the following result.

Theorem 4.4 For $A \in \mathbf{H}_{v}^{m \times n}, C \in \mathbf{H}_{v}^{p \times q}, B \in \mathbf{H}_{v}^{m \times q}$. Then

1) $\mathrm{V}$-quaternionic matrix equation $A X C=B$ has a solution if and only if $\operatorname{rank}(A)=\operatorname{rank}(A, B)$ and $\operatorname{rank}(C)=\operatorname{rank}\left(\begin{array}{l}C \\ B\end{array}\right)$, i.e. $A X C=B$ has a solution if and only if $A^{R} Y C^{R}=B^{R}$ has a solution, and if $\operatorname{rank}(A)=\operatorname{rank}(A, B)=n$, $\operatorname{rank}(C)=\operatorname{rank}\left(\begin{array}{l}C \\ B\end{array}\right)=p$, then v-quaternionic matrix equation $A X C=B$ has a unique solution.

2) If $Y$ is a solution to $A^{R} Y C^{R}=B^{R}$, then the following v-quaternion matrix is a solution to $A X C=B$,

$$
X=\frac{1}{16}\left[I_{n}, I_{n} \mathrm{i}, I_{n} \mathrm{j}, I_{n} \mathrm{k}\right]\left(Y-P_{n} Y P_{p}+\frac{1}{v} R_{n} Y R_{p}+\frac{1}{v} S_{n} Y S_{p}\right)\left[\begin{array}{c}
I_{p} \\
-I_{p} \mathrm{i} \\
\frac{1}{v} I_{p} \mathrm{j} \\
\frac{1}{v} I_{p} \mathrm{k}
\end{array}\right] .
$$

The proof process is similar to the Theorem 4.1.

Remark 2 The above theorems and corollaries not only give the necessary and sufficient conditions for quaternion and split quaternion matrix equations $A X=B, A X C=B$ to have a solution, but also a unification of representation for a solution.

\section{Example 4.1}

For two v-quaternion matrices $A$ and $B$ in Example 3.1, find solutions of the v-quaternionic linear equations $A X=B$.

By the real representation of the $\mathrm{v}$-quaternion matrix, we know 


$$
A^{R}=\left[\begin{array}{cccccccc}
0 & 1 & -1 & 0 & 0 & v & 0 & 0 \\
-1 & 0 & 0 & 0 & v & 0 & 0 & -v \\
1 & 0 & 0 & 1 & 0 & 0 & 0 & -v \\
0 & 0 & -1 & 0 & 0 & -v & -v & 0 \\
0 & 1 & 0 & 0 & 0 & 1 & -1 & 0 \\
1 & 0 & 0 & -1 & -1 & 0 & 0 & 0 \\
0 & 0 & 0 & -1 & 1 & 0 & 0 & 1 \\
0 & -1 & -1 & 0 & 0 & 0 & -1 & 0
\end{array}\right], B^{R}=\left[\begin{array}{cccc}
0 & -1 & 0 & 0 \\
-1 & 0 & 0 & 0 \\
1 & 0 & 0 & 0 \\
0 & -1 & 0 & 0 \\
0 & 0 & 0 & -1 \\
0 & 0 & -1 & 0 \\
0 & 0 & 1 & 0 \\
0 & 0 & 0 & -1
\end{array}\right],
$$

and if $v \neq \frac{7 \pm \sqrt{45}}{2}$, then $\operatorname{rank}\left(A^{R}\right)=\operatorname{rank}\left(A^{R}, B^{R}\right)=8$, i.e.

$\operatorname{rank}(A)=\operatorname{rank}(A, B)=2$, then the v-quaternionic linear equations $A X=B$ have a unique solution.

For the matrix equation $A^{R} Y=B^{R}$, the unique solution is easily found to be

$$
Y=\frac{1}{v^{2}-7 v+1}\left[\begin{array}{cccc}
-5 v+1 & 0 & (1-2 v) v & 0 \\
0 & 3 v & 0 & (-1-v) v \\
0 & -5 v+1 & 0 & (2 v-1) v \\
-3 v & 0 & (-1-v) v & 0 \\
1-2 v & 0 & -5 v+1 & 0 \\
0 & -1-v & 0 & 3 v \\
0 & 2 v-1 & 0 & -5 v+1 \\
-1-v & 0 & -3 v & 0
\end{array}\right]
$$

By (4.1), we easily find the unique solution $X$ of v-quaternionic linear equations $A X=B$, and

$$
\begin{aligned}
X & =\frac{1}{16}\left[I_{2}, I_{2} \mathrm{i}, I_{2} \mathrm{j}, I_{2} \mathrm{k}\right]\left(Y-P_{n} Y P_{p}+\frac{1}{v} R_{n} Y R_{p}+\frac{1}{v} S_{n} Y S_{p}\right)\left[\begin{array}{c}
1 \\
-\mathrm{i} \\
\frac{1}{v} \mathrm{j} \\
\frac{1}{v} \mathrm{k}
\end{array}\right] \\
& =\left[\frac{-5 v+1}{v^{2}-7 v+1}+\frac{1-2 v}{v^{2}-7 v+1} \mathrm{j} \frac{-3 v}{v^{2}-7 v+1} \mathrm{i}-\frac{1+v}{v^{2}-7 v+1} \mathrm{k}\right]^{\mathrm{T}} .
\end{aligned}
$$

The following two examples are special cases of the above conclusion.

Case 1: For quaternionic linear equations $A X=B$ with $v=-1$. It is easy to know $A^{R}$ and $B^{R}$ by (2.6a),

$$
A^{R}=\left[\begin{array}{cccccccc}
0 & 1 & -1 & 0 & 0 & -1 & 0 & 0 \\
-1 & 0 & 0 & 0 & -1 & 0 & 0 & 1 \\
1 & 0 & 0 & 1 & 0 & 0 & 0 & 1 \\
0 & 0 & -1 & 0 & 0 & 1 & 1 & 0 \\
0 & 1 & 0 & 0 & 0 & 1 & -1 & 0 \\
1 & 0 & 0 & -1 & -1 & 0 & 0 & 0 \\
0 & 0 & 0 & -1 & 1 & 0 & 0 & 1 \\
0 & -1 & -1 & 0 & 0 & 0 & -1 & 0
\end{array}\right], B^{R}=\left[\begin{array}{cccc}
0 & -1 & 0 & 0 \\
-1 & 0 & 0 & 0 \\
1 & 0 & 0 & 0 \\
0 & -1 & 0 & 0 \\
0 & 0 & 0 & -1 \\
0 & 0 & -1 & 0 \\
0 & 0 & 1 & 0 \\
0 & 0 & 0 & -1
\end{array}\right],
$$


and $\operatorname{rank}\left(A^{R}\right)=\operatorname{rank}\left(A^{R}, B^{C}\right)=8$. Clearly, the linear equations $A^{R} Y=B^{R}$ have a unique solution. The unique solution is easily found to be

$$
Y=\frac{1}{9}\left[\begin{array}{cccc}
6 & 0 & -3 & 0 \\
0 & -3 & 0 & 0 \\
0 & 6 & 0 & 3 \\
3 & 0 & 0 & 0 \\
3 & 0 & 6 & 0 \\
0 & 0 & 0 & -3 \\
0 & -3 & 0 & 6 \\
0 & 0 & 3 & 0
\end{array}\right]
$$

By (4.8), we easily find the unique solution $X$ of quaternionic linear equations $A X=B$, and

$$
\begin{aligned}
& X=\frac{1}{16}\left[I_{2}, I_{2} \mathrm{i}, I_{2} \mathrm{j}, I_{2} \mathrm{k}\right]\left(Y-P_{n} Y P_{p}-R_{n} Y R_{p}-S_{n} Y S_{p}\right)\left[\begin{array}{c}
1 \\
-\mathrm{i} \\
-\mathrm{j} \\
-\mathrm{k}
\end{array}\right] \\
& =\left[\begin{array}{ll}
\frac{2}{3}+\frac{1}{3} \mathrm{j} & \frac{1}{3} \mathrm{i}
\end{array}\right]^{\mathrm{T}} .
\end{aligned}
$$

Case 2: For split quaternionic linear equations $A X=B$ with $v=1$. It is easy to know $A^{R}$ and $B^{R}$ by (2.6b),

$$
A^{R}=\left[\begin{array}{cccccccc}
0 & 1 & -1 & 0 & 0 & 1 & 0 & 0 \\
-1 & 0 & 0 & 0 & 1 & 0 & 0 & -1 \\
1 & 0 & 0 & 1 & 0 & 0 & 0 & -1 \\
0 & 0 & -1 & 0 & 0 & -1 & -1 & 0 \\
0 & 1 & 0 & 0 & 0 & 1 & -1 & 0 \\
1 & 0 & 0 & -1 & -1 & 0 & 0 & 0 \\
0 & 0 & 0 & -1 & 1 & 0 & 0 & 1 \\
0 & -1 & -1 & 0 & 0 & 0 & -1 & 0
\end{array}\right], B^{R}=\left[\begin{array}{cccc}
0 & -1 & 0 & 0 \\
-1 & 0 & 0 & 0 \\
1 & 0 & 0 & 0 \\
0 & -1 & 0 & 0 \\
0 & 0 & 0 & -1 \\
0 & 0 & -1 & 0 \\
0 & 0 & 1 & 0 \\
0 & 0 & 0 & -1
\end{array}\right]
$$

and $\operatorname{rank}\left(A^{R}\right)=\operatorname{rank}\left(A^{R}, B^{R}\right)=8$. Clearly, the linear equations $A^{R} Y=B^{R}$ have a unique solution. The unique solution is easily found to be

$$
Y=-\frac{1}{5}\left[\begin{array}{cccc}
-4 & 0 & -1 & 0 \\
0 & 3 & 0 & -2 \\
0 & -4 & 0 & 1 \\
-3 & 0 & -2 & 0 \\
-1 & 0 & -4 & 0 \\
0 & -2 & 0 & 3 \\
0 & 1 & 0 & -4 \\
-2 & 0 & -3 & 0
\end{array}\right]
$$

By (4.9), we easily find the unique solution $X$ of split quaternionic linear equations $A X=B$, and 


$$
\begin{aligned}
X & =\frac{1}{16}\left[I_{2}, I_{2} \mathrm{i}, I_{2} \mathrm{j}, I_{2} \mathrm{k}\right]\left(Y-P_{n} Y P_{p}+R_{n} Y R_{p}+S_{n} Y S_{p}\right)\left[\begin{array}{c}
1 \\
-\mathrm{i} \\
\mathrm{j} \\
\mathrm{k}
\end{array}\right] \\
& =\left[\frac{4}{5}+\frac{1}{5} \mathrm{j} \frac{3}{5} \mathrm{i}+\frac{2}{5} \mathrm{k}\right]^{\mathrm{T}} .
\end{aligned}
$$

\section{Conclusion}

The goal of this paper is to solve the quaternion and split quaternion linear equations in a unified manner. First, we give the definition of the v-quaternion and two new matrix representations of v-quaternion matrix. Then we derive two algebraic methods for solving the linear equations of v-quaternion. It is noteworthy that this paper not only gives algebraic techniques for solving the linear equations over v-quaternion algebras, but also a unification of algebraic techniques for linear equations in quaternionic and split quaternionic theory.

\section{Acknowledgements}

We thank the Editor and the referee for their comments. Research of T. Jiang is funded by the National Natural Science Foundation of China (11771188) and Shandong Natural Science Foundation (ZR201709250116). This support is greatly appreciated.

\section{Conflicts of Interest}

The authors declare no conflicts of interest regarding the publication of this paper.

\section{References}

[1] Hamilton, W.R. (1843) On a New Species of Imaginary Quantities Connected with a Theory of Quaternions. Proceedings of the Royal Irish Academy, 2, 424-434.

[2] Özdemir, M. and Ergin, A.A. (2006) Rotations with Unit Timelike Quaternions in Minkowski 3-Space. Journal of Geometry and Physics, 56, 322-336. https://doi.org/10.1016/j.geomphys.2005.02.004

[3] Schmidt, J. and Nieman, H. (2001) Using Quaternions for Parametrizing 3-D Rotations in Unconstrained Nonlinear Optimization. Vision Modeling, and Visualization, Stuttgart, 21-23 November 2001, 399-406.

[4] Cockle, J. (1849) On Systems of Algebra Involving More than One Imaginary and on Equations of the Fifth Degree. Philosophical Magazine, 35, 434-437. https://doi.org/10.1080/14786444908646384

[5] Kula, L. and Yayli, Y. (2007) Split Quaternions and Rotations in Semi Euclidean Space $\mathrm{E}_{2}^{4}$. Journal of the Korean Mathematical Society, 44, 1313-1327. https://doi.org/10.4134/JKMS.2007.44.6.1313

[6] Özkaldi, S. and Gündoğan, H. (2011) Dual Split Quaternions and Screw Motion in 3-Dimensional Lorentzian Space. Advances in Applied Clifford Algebras, 21, 193-202. https://doi.org/10.1007/s00006-010-0236-6 
[7] Özdemir, M., Erdoğdu, M. and Simsek, H. (2014) On the Eigenvalues and Eigenvectors of a Lorentzian Rotation Matrix by Using Split Quaternions. Advances in Applied Clifford Algebras, 24, 179-192. https://doi.org/10.1007/s00006-013-0424-2

[8] Adler, S.L. (1995) Quaternionic Quantum Mechanics and Quantum Fields. Oxford University Press, New York.

[9] Özdemir, M. and Ergin, A.A. (2005) Some Geometric Applications of Timelike Quaternions. International Conference of the Jangjeon Mathematical Society, Vol. 16, 108-115.

[10] Ramis, C. and Yayli, Y. (2013) Dual Split Quaternions and Chasles' Theorem in 3-Dimensional Minkowski Space $\mathrm{E}_{1}^{3}$. Advances in Applied Clifford Algebras, 23, 951-964. https://doi.org/10.1007/s00006-013-0405-5

[11] Aslan, S. and Yayli, Y. (2016) Split Quaternions and Canal Surfaces in Minkowski 3-Space. International Journal of Geometry, 5, 51-61.

[12] Brody, D.C. and Graefe, E.M. (2011) On Complexified Mechanics and Coquaternions. Journal of Physics A: Mathematical and Theoretical, 44, Article ID: 072001. https://doi.org/10.1088/1751-8113/44/7/072001

[13] Janovská, D. and Opfer, G. (2013) Linear Equations and the Kronecker Product in Coquaternions. Mitteilungen der Mathematische Gesellschaft in Hamburg, 33, 181-196.

[14] Jiang, T. (2004) An Algorithm for Quaternionic Linear Equations in Quaternionic Quantum Theory. Journal of Mathematical Physics, 45, 4218-4222. https://doi.org/10.1063/1.1794368

[15] Zhang, Z., Jiang, Z. and Jiang, T. (2015) Algebraic Methods for Least Squares Problem in Split Quaternionic Mechanics. Applied Mathematics and Computation, 269, 618-625. https://doi.org/10.1016/j.amc.2015.07.072 